\title{
Survival probability and local density of states for one-dimensional Hamiltonian systems
}

\author{
Jiří Vaníček ${ }^{1,2}$ and Doron Cohen ${ }^{3}$ \\ ${ }^{1}$ Department of Physics, Harvard University, Cambridge, MA 02138 \\ 2 Mathematical Sciences Research Institute, Berkeley, CA 94720 \\ ${ }^{3}$ Department of Physics, Ben-Gurion University, Beer-Sheva 84105, Israel
}

\begin{abstract}
For chaotic systems there is a theory for the decay of the survival probability, and for the parametric dependence of the local density of states. This theory leads to the distinction between "perturbative" and "non-perturbative" regimes, and to the observation that semiclassical tools are useful in the latter case. We discuss what is "left" from this theory in the case of one-dimensional systems. We demonstrate that the remarkably accurate uniform semiclassical approximation captures the physics of all the different regimes, though it cannot take into account the effect of strong localization.
\end{abstract}

\section{Introduction}

The quantum mechanical state of a particle, or of a system, is represented by the probability matrix $\rho$. This object corresponds to a classical distribution $\rho^{c l}(Q, P)$ in phase space, where $Q$ and $P$ are the canonical coordinates of the system. One way to represent the probability matrix is by using the Wigner function $\rho(Q, P)$. Many calculations in quantum mechanics reduce eventually to calculation of a trace over a pair of probability matrices. This includes in particular calculations of the local density of states (LDOS) [1, and calculations of the survival probability $\mathcal{P}(t)$ 2. Strongly related are calculations of Franck-Condon factors for non-adiabatic transitions between Born-Oppenheimer surfaces [3. Lately [4, 5, 6, 7] there is a special interest in calculation of "fidelity" (also known as "Loschmidt echo") in the context of quantum computation. This "fidelity" is in fact a synonym for a "survival probability" $\mathcal{P}(t)$ which is calculated for a particular dynamical scenario (namely, the forward evolution is followed by a reversed evolution with a perturbed Hamiltonian).

The calculation of a trace over a pair of probability matrices has a clear classical limit. To be specific let us consider the survival probability, which is defined as

$$
\mathcal{P}(t)=\left|\left\langle\psi_{0} \mid \psi_{t}\right\rangle\right|^{2}=\operatorname{trace}\left(\rho_{t} \rho_{0}\right) .
$$

where $\rho_{t}$ and $\psi_{t}$ are the probability matrix and the associated wavefunction of an evolving quantum mechanical pure state. In the Wigner representation the trace operation means $d Q d P /(2 \pi \hbar)^{d}$ integral over phase space, where $d$ is the number of freedoms. It should be emphasized that the Wigner representation is quantummechanically exact. The classical limit/approximation is obtained by treating $\rho(P, Q)$ as a classical distribution, whose evolution is governed by classical equations of motion.

To take the classical limit as a leading order approximation for $\mathcal{P}(t)$ is one possibility. Another possibility is to use perturbation theory, in particular "Fermi 
golden rule" (FGR). It should be obvious that the results that are obtained by using these methods are typically very different from each other.

A major objective of recent studies 8 is to understand the limitations of perturbation theory on the one hand, and to explore the capabilities of the semiclassical tools, on the other. A specific question is whether the decay of $\mathcal{P}(t)$ is of perturbative nature (e.g. FGR type $\ddagger$ ), or else whether it is of classical nature ("semiclassical" type). The main approach towards this question is to allow the specification of a control parameter that represents the "strength" of a perturbation. Depending on the value of of this control parameter one may have either perturbative or semiclassical behavior. The first publications [1] 1] that have taken this approach, led to the distinction between "perturbative" and "non-perturbative" regimes, and to the realization that the "semiclassical" behavior is contained in the" non-perturbative" regime. Later [5] the idea was adopted into the context of "fidelity" studies.

The above mentioned studies have mainly concentrated on quantized chaotic systems. The chaos assumption allows simplification of certain calculations. In particular one can invoke the random matrix theory (RMT) conjecture, in order to obtain some "generic" results. The natural question that arises is whether some of the general theory regarding the LDOS and $\mathcal{P}(t)$, applies also to the world of one dimensional $(d=1)$ systems.

A central observation in the theory of quantized chaotic systems is the existence of two distinct energy scales. One is the mean level spacing $\left(\Delta \propto \hbar^{d}\right)$, while the other is the bandwidth $\left(\Delta_{b} \propto \hbar\right)$. The latter is semiclassically related to the correlation time of the classical motion. The dimensionless bandwidth is defined as $b=\Delta_{b} / \Delta$. The classical limit $(\hbar \rightarrow 0)$ of quantized chaotic system $(d>1)$ is characterized by $\Delta \ll \Delta_{b}$ and hence $b \gg 1$. But in one dimensional systems $(d=1)$ we do not necessarily have this separation of energy scales. For some typical perturbations $b=\mathcal{O}(1)$. We shall explain that in such case there is no FGR regime in the theory. A necessary, but not sufficient condition for having FGR regime in one-dimensional systems is to have $b \gg 1$. This means that "small features" should characterize the phase space manifolds that support the perturbed (or evolving) quantum mechanical states.

The analysis of one dimensional $(d=1)$ systems is highly non-universal, but typically allows analytical calculations that go well beyond the leading semiclassical approximation. In particular we demonstrate the capabilities and the limitations of the uniform approximation [12, 13].

The outline of this paper is as follows: In Section 2 we define some simple prototype models. In Section 3 we define the main objects of the studies which are the LDOS and the survival probability $\mathcal{P}(t)$. In Section 4 we discuss the issue of quantumclassical correspondence (QCC) and make the distinction between "semiclassical" and "perturbative" approximations. In Sections [5]and [6] we discuss the notion of "regimes" in the context of LDOS studies. In Sections 7 and 8 we discuss the existence of LDOS regimes in 1D within the framework of a uniform approximation. In Section 9 we discuss the implication of the strong localization effect. In Section 10 we extend the general consideration into the context of survival probability studies. Conclusion and final remarks are summarized in Section [1] The appendices are an integral part of the Paper. They contain details of derivations and have not been integrated into the main text in order to simplify the presentation of the physical picture.

‡ In section 5 we explain that perturbation theory can lead to either no decay, or Gaussian decay, or Wigner type decay. The latter can be regarded as the outcome of FGR transitions. 


\section{Definition of the models}

In this paper we are going to consider one-dimensional model systems. The simplest is the deformable harmonic oscillator:

$$
\mathcal{H}(Q, P ; x)=\frac{1}{2}(x P)^{2}+\frac{1}{2}(Q / x)^{2} .
$$

where $(Q, P)$ are the canonical coordinates, and $x$ is a constant parameter. The energy surfaces $\mathcal{H}(Q, P ; x)=E$ are ellipses in phase space. We shall assume that $E \gg 1$. Without loss of generality we shall regard $x=x_{0}=1$ as the "unperturbed" value of the parameter $x$. Later we shall assume that $\delta x \equiv\left(x-x_{0}\right)$ is small in a classical sense. Namely $\delta x \ll 1$. Then it is possible to write the Hamiltonian as $\mathcal{H}=\mathcal{H}_{0}+\delta \mathcal{H}$, where

$$
\begin{aligned}
\mathcal{H}_{0} & =\mathcal{H}\left(Q, P ; x_{0}=1\right)=\frac{1}{2}\left(P^{2}+Q^{2}\right), \\
\delta \mathcal{H} & =\left.\delta x \frac{\partial \mathcal{H}}{\partial x}\right|_{x=1}=\delta x\left(P^{2}-Q^{2}\right)
\end{aligned}
$$

Note that the perturbation $\delta x$ is allowed to be large in a quantum mechanical sense: many levels can be "mixed" by the perturbation $\delta \mathcal{H}$.

A more complicated case is to consider a particle in a ring (= one dimensional box with periodic boundary conditions). The Hamiltonian is of the general form

$$
\mathcal{H}(Q, P ; x)=\frac{1}{2 \mathrm{~m}} P^{2}+x V(Q)
$$

Without loss of generality we can take $\mathrm{m}=1$ as the mass of the particle, and $L=2 \pi$ as the perimeter (length) of the ring. The parameter $x$ controls the "height" of the potential landscape. Naturally we shall regard $x_{0}=0$ as the unperturbed value of $x$. We shall consider the case of a single "bump" where

$$
V(Q)=V_{0} \exp \left[-\left(Q-Q_{0}\right)^{2} / 2 \ell^{2}\right]
$$

It is implicitly assumed that $\ell \ll L$. The Fourier components of this potential are $|\tilde{V}(k)|=V_{0} \ell \exp \left[-(k \ell)^{2} / 2\right]$. Hence the non-vanishing $(|k \ell|<1)$ Fourier components satisfy $|\tilde{V}(k)| \approx V_{0} \ell$. If we have many bumps, then we have a "disordered" potential

$$
V(Q)=\sum_{\alpha}( \pm \text { random }) V_{0} \exp \left[-\left(Q-Q_{\alpha}\right)^{2} / 2 \ell^{2}\right]
$$

Here we implicitly assume that the bumps are non-overlapping and randomly distributed along the ring, such that the correlation function $\langle V(Q+r) V(Q)\rangle$ is characterized by the correlation length $\ell$. Consequently the non-vanishing $(|k \ell|<1)$ Fourier components of the potential satisfy $|\tilde{V}(k)| \approx V_{0} \ell \times \sqrt{L / \ell}$. Note that the phases of the Fourier components look "random", unlike the case of a single bump.

All models that we have introduced are of the generic form $\mathcal{H}=\mathcal{H}_{0}+\delta \mathcal{H}$. The representation in the basis that is determined by the unperturbed Hamiltonian is

$$
\mathcal{H} \mapsto \mathbf{E}+\delta x \mathbf{B}
$$

where $\mathbf{E}=\left\{E_{n}\right\}$ is a diagonal matrix consisting of the eigenenergies of the unperturbed Hamiltonian. With scaled parameters, such that $\hbar=1$, the mean level spacing of the eigenenergies is $\Delta=1$ for Hamiltonian (2) and $\Delta=\sqrt{2 E}$ for Hamiltonian (5). The matrix $\mathbf{B}$ corresponds to the perturbation. It is a banded matrix. The bandwidth is $b$. It is defined such that $2 b$ is the number of the levels which are coupled by the perturbation. For the Hamiltonian (2) we have $b=1$, because only neighboring levels of the de-symmetrized Hamiltonian $(|n-m|=2)$ are coupled by the perturbation $\delta \mathcal{H}$. For the Hamiltonian (5) the bandwidth is $b=L / \ell$. See Section $[$ for details. Thus for the latter model we may have $b \gg 1$. 


\section{Definitions of $P(n \mid m)$ and $\mathcal{P}(t)$}

Let $|n(x)\rangle$ and $E_{n}(x)$ be the eigenstates and the corresponding eigenvalues of the Hamiltonian $\mathcal{H}(Q, P ; x)$. We define the "parametric" kernel

$$
P(n \mid m)=\left|\left\langle n(x) \mid m\left(x_{0}\right)\right\rangle\right|^{2}=\operatorname{trace}\left(\rho_{n} \rho_{m}\right) .
$$

Note that for $\delta x=0$ we have $P(n \mid m)=\delta_{n m}$. Given a reference state $\left|m\left(x_{0}\right)\right\rangle$ this kernel can be regarded as a probability distribution with respect to $n$. The LDOS is just a scaled version of this kernel, namely

$$
P(\omega)=\sum_{n} P(n \mid m) 2 \pi \delta\left(\omega-\left[E_{n}(x)-E_{m}\left(x_{0}\right)\right]\right)
$$

One important measure that characterizes the LDOS is its variance:

$$
\delta E^{2}=\sum_{n} P(n \mid m)\left(E_{n}-E_{m}\right)^{2}
$$

In the next section we shall explain that the variance has a special role in the theory of the LDOS.

The survival probability of the state $\left|m\left(x_{0}\right)\right\rangle$ for evolution which is generated by the perturbed Hamiltonian $\mathcal{H}(Q, P ; x)$ can be written as $\mathcal{P}(t)=|F(t)|^{2}$, where

$$
F(t)=\left\langle m\left(x_{0}\right)\left|\mathrm{e}^{-i t\left(\mathcal{H}-E_{m}\right)}\right| m\left(x_{0}\right)\right\rangle
$$

In the above definition we have taken $E=E_{m}$ as the natural reference for the energy. With this definition we see that $F(t)$ is just the Fourier transform of the LDOS. We note that in more complicated scenarios a simple Fourier transform relation between $F(t)$ and $P(\omega)$ does not exist $\underline{6}$.

\section{Quantal-classical correspondence (QCC)}

The classical limit/approximation for the LDOS kernel $P(n \mid m)$ is obtained by taking for the Wigner function $\rho_{n}(Q, P) \approx \rho_{n}^{c l}(Q, P)$, where $\rho_{n}^{c l}(Q, P) \propto \delta\left[\mathcal{H}(Q, P ; x)-E_{n}(x)\right]$ is the corresponding classical microcanonical distribution. For the deformable harmonic oscillator one obtains Appendix A:

$$
P^{c l}(n \mid m) \approx \frac{1}{\pi} \frac{1}{\sqrt{4(\delta x / x)^{2} E^{2}-\left(E_{n}-E_{m}\right)^{2}}}
$$

The dispersion (square root of the variance) is

$$
\delta E^{c l}=\sqrt{2}\left(\frac{\delta x}{x}\right) E
$$

What about the quantum mechanical LDOS? The simplest limit that can be considered is first order perturbation theory (FOPT). One obtains Appendix B):

$$
P^{\mathrm{prt}}(n \mid m) \approx \delta_{n m}+\frac{1}{4}\left(\frac{\delta x}{x} E\right)^{2} \delta_{|n-m|, 2} \quad[\mathrm{FOPT}]
$$

This result is very different from the classical result. Therefore we say that there is no "detailed QCC". On the other hand one easily calculates the variance. One obtains $\delta E=\delta E^{c l}$. We call the latter equality "restricted QCC".

It is possible to write an exact analytical expression for the quantum mechanical LDOS (see Appendix A). But this expression is not very useful since its complexity 
makes it virtually impossible to extract the simple limits in various regimes. It is more illuminating to obtain a uniform approximation Appendix C

$$
P^{\text {uniform }}(n \mid m) \approx\left[J_{(m-n) / 2}\left(\frac{\delta x}{x} E\right)\right]^{2}
$$

In figure पwe demonstrate that the uniform approximation is almost indistinguishable from the exact result for any value of $\delta x$. One can verify that this approximation reduces to the FOPT result (15) in the parametric regime $\delta x \ll(x / E)$. Disregarding an oscillatory component it reduces to the semiclassical result (13) in the parametric regime $\delta x \gg(x / E)$. The two regimes are separated by the quantum mechanical parametric scale $\delta x_{\mathrm{prt}}=\left(\hbar \omega_{\text {osc }} / E\right) x$. For the convenience of the reader we have reverted here to non-scaled units (with our scaling $\omega_{\text {osc }}=1$ and $\hbar=1$.)

The example above demonstrates some general observations regarding the LDOS. On the one hand we have restricted QCC, which means $\delta E=\delta E^{c l}$. It can be proved that this type of QCC holds in general [14]. The proportionality $\delta E \propto \delta x$ is guaranteed by the classical linearization condition, which is a fixed assumption in this paper (in the present example it means $\delta x \ll x)$. On the other hand we do not have in general detailed QCC, which means that the approximation $P(n \mid m) \approx P^{c l}(n \mid m)$ holds only in a specific parametric regime. In the above example we have only two parametric regimes:

- The "perturbative regime" $\left(\delta x \ll \delta x_{\mathrm{prt}}\right)$

in which (in this example) FOPT can be used.

- The "non-perturbative regime" $\left(\delta x>\delta x_{\mathrm{prt}}\right)$

in which (in this example) the semiclassical approximation can be used.

Hence upon quantization there is only one parametric scale in the theory $\left(\delta x_{\mathrm{prt}} \propto \hbar\right)$. This parametric scale marks a border between the perturbative and the nonperturbative regimes.

\section{Regimes in case of chaotic systems}

In case of chaotic systems the generic case is having three regimes. The "perturbative regime" is subdivided into a "FOPT regime" and a Wigner/Lorentzian/core-tail/FGR regime. In the latter case the various names mean the same, and we shall use from now on the term "Wigner regime". The FOPT regime is defined as the parametric regime where we can use first order perturbation theory (FOPT):

$$
P^{\text {prt }}(n \mid m) \approx \delta_{n m}+\delta x^{2} \frac{\left|\mathbf{B}_{n m}\right|^{2}}{\left(E_{n}-E_{m}\right)^{2}} \quad[\mathrm{FOPT}]
$$

The condition for the validity of this approximation is $\delta x \ll \delta x_{c}$, where $\delta x_{c}=\Delta / \sigma$. Here $\Delta$ is the mean level spacing, and $\sigma$ is defined as the RMS value of the "in-band" off-diagonal elements of the $\mathbf{B}$ matrix.

Outside of the "FOPT regime" we can still use first order perturbation theory for the tails of the LDOS [10]. This leads to a generalized Wigner's Lorentzian (a more meaningful name is "core-tail structure"):

$$
P^{\text {prt }}(n \mid m) \approx \delta x^{2} \frac{\left|\mathbf{B}_{n m}\right|^{2}}{[\Gamma(\delta x) / 2]^{2}+\left(E_{n}-E_{m}\right)^{2}} \quad \text { [Wigner] }
$$

The width parameter $\Gamma(\delta x)$ is determined so as to have proper normalization $\left(\sum_{n} P(n \mid m)=1\right)$. For strongly chaotic systems, for which the band profile is quite 

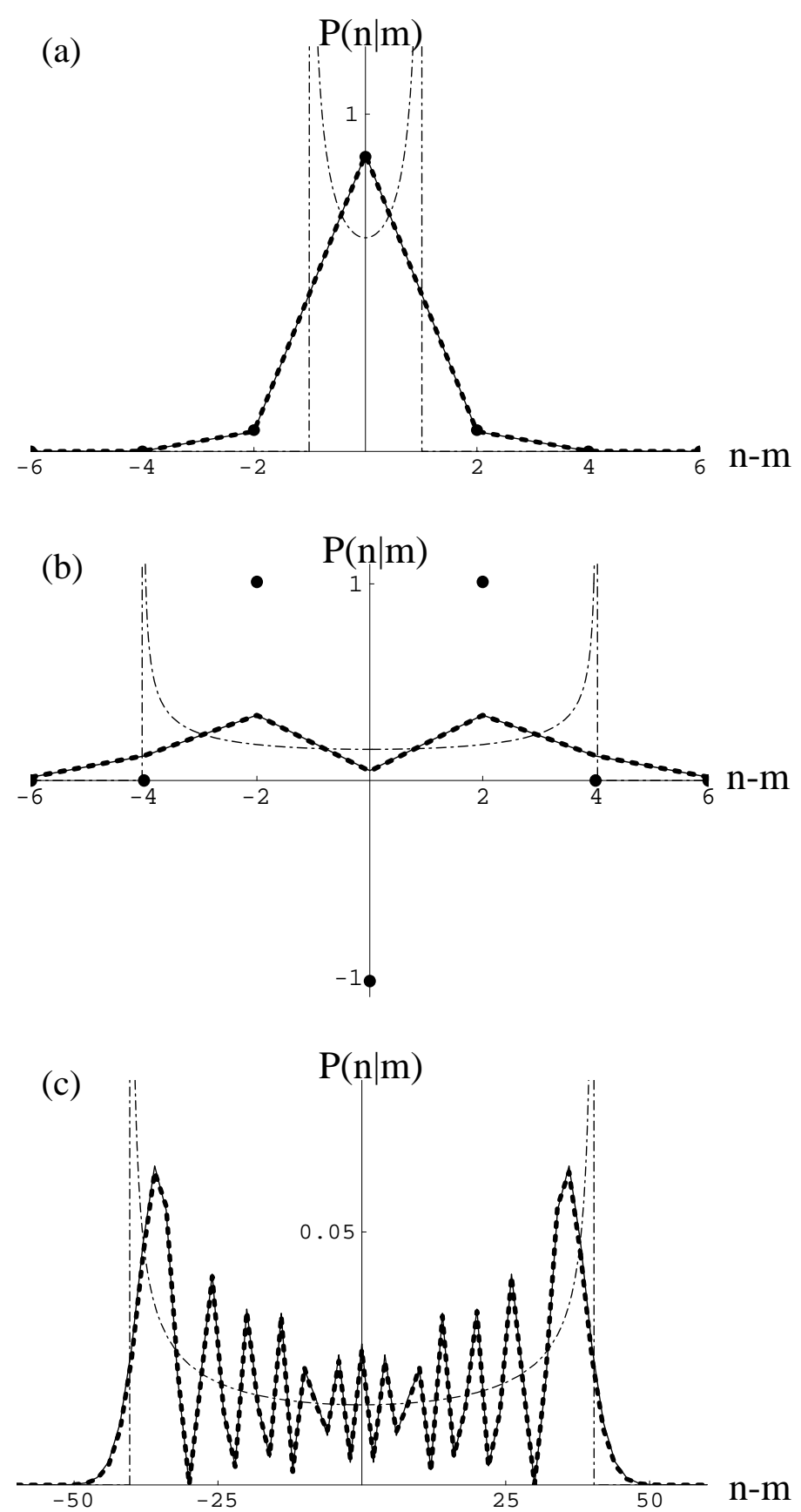

Figure 1. The local density of states for $\mathrm{E}=100$. (a) For $(\delta x / x) E=0.5$ (perturbative regime). (b) For $(\delta x / x) E=2$ (intermediate regime). (c) For $(\delta x / x) E=20$ (semiclassical regime). In each panel the thick dashed line is the exact result, the solid line is the uniform result, the dashed-dotted line is the classical result, and the solid circles are the perturbative result. The classical result is divided by 2 for a reason which is explained at the end of Appendix A 
flat, it follows that the "core" width is $\Gamma \approx\left(\delta x / \delta x_{c}\right)^{2} \Delta$. The "core-tail" approximation makes sense as long as we have separation of energy scales $\Gamma(\delta x) \ll \Delta_{b}$, where $\Delta_{b}=b \Delta$ is the bandwidth in units of energy. This translates into the condition $\delta x \ll \delta x_{\text {prt }}$ where

$$
\delta x_{\mathrm{prt}}=\sqrt{b} \delta x_{c} .
$$

The "core-tail" approximation (18) can be regarded as the outcome of perturbative summation of diagrams to infinite order: The FOPT diagrams are reiterated, while the interference terms are neglected. In time domain analysis this corresponds to a Markovian approximation for the survival probability $\mathcal{P}(t)$, leading to an exponential decay. Hence we say that there is a perturbative regime that includes the FOPT sub-regime, and the Wigner sub-regime. In the Wigner sub-regime we need all orders of perturbation theory leading to FGR transitions and Wigner decay. On the other hand in the FOPT sub-regime there is no decay. This can be easily deduced by Fourier transforming the LDOS which is associated with Eq.(17): Up to negligible first order correction, the survival amplitude $F(t)$ comes out as a pure phase factor, whose absolute value squared is $\mathcal{P}(t) \approx 1$. It is appropriate at this point to make a connection with recent fidelity studies [4]. There, it is customary to consider the dynamics of a wavepacket that is a superposition of many eigenstates. Consequently, in fidelity studies, there is an effective averaging over the survival amplitude $F(t)$, leading (in the FOPT regime) to a slow Gaussian decay which is trivially related to the statistics of the first order correction to the eigenenergies.

We can summarize this section by stating that in case of a generic quantized chaotic system we have three regimes as follows:

- The FOPT regime $\left(\delta x \ll \delta x_{c}\right)$ where Eq. (17) can be trusted.

- The Wigner (perturbative) regime $\left(\delta x_{c} \ll \delta x \ll \delta x_{\text {prt }}\right)$, where Eq. (18) can be trusted.

- The non-perturbative regime $\left(\delta x>\delta x_{\mathrm{prt}}\right)$, in which (typically) the semiclassical approximation can be used.

Hence upon quantization there are two parametric scales in the theory, which are $\delta x_{c}$ and $\delta x_{\mathrm{prt}} \propto \hbar$. These parametric scale mark the borders between the regimes.

\section{Regimes in case of $1 \mathrm{D}$ systems}

By Weyl law we know that the mean level spacing for $d$-dimensional system is $\Delta \propto \hbar^{d}$. On the other hand the bandwidth $\Delta_{b} \propto \hbar$ is inversely proportional to the period, or to the correlation time of the dynamics. For chaotic systems (with $d>1$ ) we generally have $b=\Delta_{b} / \Delta \gg 1$. But for $d=1$ systems we can have $b=\mathcal{O}(1)$, as in the example of equation (2). Therefore in the latter case $\delta x_{\mathrm{prt}} \sim \delta x_{c}$, and we do not have a "Wigner regime". This means that the LDOS cannot have a Lorentzian-like structure, and consequently the survival probability $\mathcal{P}(t)=|F(t)|^{2}$ cannot have an exponential decay. While this latter statement strictly holds for the specific scenario which has been defined by equation (12), it typically holds also in more complicated circumstances ("fidelity" studies). Thus we conclude that the FGR picture cannot be applicable to the analysis of the dynamics unless $b \gg 1$.

At this stage of the discussion we can say that in order to have three regimes (FOPT, Wigner, semiclassical) in the theory of one-dimensional systems, we have to 
consider models where the perturbation is characterized by a large bandwidth $(b \gg 1)$. In the next sections we would like to further discuss the following:

- A uniform approximation can be applied in order to address both the perturbative and the non-perturbative regimes.

- We can have $b \gg 1$ by considering potentials that have small (or sharp) features. (For example "bump" or "disorder").

- It is possible to get a Lorentzian-like LDOS from the uniform approximation, but $b \gg 1$ is not a sufficient condition.

- The uniform approximation does not take into account the effect of strong localization.

- Three LDOS regimes can be observed in case of disordered potential in spite of the strong localization effect.

\section{Perturbations with large bandwidth}

For any one-dimensional system we can find the action-angle variables, in which the problem becomes essentially equivalent to the problem (5) of a particle in a ring. Consider for example the deformable harmonic oscillator (2): We already saw that all the essential physics of the perturbed eigenstates can be obtained via a uniform approximation which is based on action-angle variables description of the system. The Hamiltonian (C.3) of a deformable harmonic oscillator in action-angle variables, and the Hamiltonian (5) of a particle in a ring, are similar as far as the semiclassical treatment is concerned. Having a quadratic rather than a linear dispersion relation is not an essential difference.

More generally, we may encounter circumstances where the perturbation creates small phase space structures. This is the new ingredient (compared with the deformable harmonic oscillator) that we are going to consider in the following sections. The problem (5) of a particle in a ring constitutes a prototype example of having such small phase space structures. The perturbation $V(Q)$ is characterized by a different, much smaller scale $(\ell)$, compared with the size of the system $(L)$. This means that we have large bandwidth $(b \gg 1)$ rather than $b=\mathcal{O}(1)$.

In the absence of a perturbation $\left(x=x_{0}=0\right)$, the eigenenergies of a particle in a ring are $E_{n}=p_{n}^{2} / 2 \mathrm{~m}$, where $n$ is an integer index, and $p_{n}=(2 \pi \hbar / L) n$. The perturbation matrix is related to the Fourier components of the potential:

$$
\mathbf{B}_{n m}=\frac{1}{L} \tilde{V}\left(p_{n}-p_{m}\right)
$$

The expressions for $\tilde{V}(k)$ in case of either the "bump" (6) or the "disorder" (7), were given in Section 2 Substitution in (20) allows to determine the bandwidth:

$$
\begin{aligned}
\Delta & =2 \pi \hbar v_{E} / L \\
\Delta_{b} & =2 \pi \hbar v_{E} / \ell \\
b & =L / \ell
\end{aligned}
$$

where $v_{E}=\sqrt{2 E / \mathrm{m}}$. One difference between the two models is related to the coupling parameter $\sigma$, which has been defined in the beginning of Section 5 as the RMS value of the in-band off-diagonal elements:

$$
\begin{array}{ll}
\sigma=(\ell / L) V_{0} & \text { for the bump } \\
\sigma=(\ell / L)^{1 / 2} V_{0} & \text { for the disorder }
\end{array}
$$


This leads to

$$
\begin{aligned}
& \delta x_{c}^{\text {bump }}=\Delta / \sigma=\left(\Delta_{b} / V_{0}\right) \\
& \delta x_{c}^{\text {disorder }}=\Delta / \sigma=\left(\Delta_{b} / V_{0}\right) / \sqrt{b}
\end{aligned}
$$

However, the difference in $\sigma$, and hence in $\delta x_{c}$ is not the significant difference between the "bump" potential and the "disorder" potential. The significant difference is related to the statistical properties of the $\mathbf{B}$ matrix. In the case of "disorder" the matrix elements look "random", which is not the case for a single bump.

In both cases, of having either single bump or disorder, the FOPT regime is $\delta x \ll \delta x_{c}$. What do we have beyond FOPT? More specifically, the question is whether we have, as in the theory of chaotic systems, a distinct parametric scale $\delta x_{p r t}=\sqrt{b} \delta x_{c}$ that distinguishes between a "Wigner regime" and a "semiclassical regime". In the next section we shall try to address this question within the framework of the uniform approximation.

\section{Regimes within the framework of the uniform approximation}

There is a general semiclassical procedure that associates wavefunctions of integrable systems with phase space manifolds. The traditional implementation of this procedure, in case of one dimensional systems, is known as "the WKB method". The WKB method is problematic near turning points. This problem can be solved by "uniformization" of the solution. The simplest point of view regarding this "uniformization" is obtained by using action-angle variables. This leads to a Hamiltonian that looks like that of a particle in a ring. In general the dispersion relation can be different (not quadratic as in (5D), but we shall see that this is not an important difference for the issues under study.

The eigenstates of Hamiltonian (5) are supported by the manifolds $H(Q, P ; x)=$ $E_{n}$. An alternate (explicit) way to describe a given manifold is $P=p_{n}(Q ; x)$, where

$$
p_{n}(Q ; x)=\sqrt{2 \mathrm{~m}\left[E_{n}-\delta x V(Q)\right]} \approx p_{n}-\delta x \hbar k(Q)
$$

and $k(Q)=V(Q) / \hbar v_{E}$. In the following we use units such that $\hbar=1$. The semiclassical formula for the corresponding eigenfunction is

$$
\langle Q \mid n(x)\rangle=\frac{1}{\sqrt{L}} \exp \left(i \int_{0}^{Q} p_{n}\left(Q^{\prime} ; x\right) d Q^{\prime}\right)
$$

This can be regarded as a special case of C.5. Above we approximate the classical pre-exponential prefactor by $1 / \sqrt{L}$. This is legitimate because a fixed assumption of this Paper is that we are considering high lying eigenstates, and assume classically small perturbations $\S$. We already saw, in the context of the deformable harmonic oscillator (see the end of Appendix C), that the numerical error which is associated with this approximation is insignificant. The essential physics of having various "regimes" is not related to this approximation.

The overlap of the semiclassical wavefunctions is given by the integral

$$
\left\langle n(x) \mid m\left(x_{0}\right)\right\rangle=\frac{1}{L} \int_{0}^{L} d Q \exp \left[-i\left(\left(p_{n}-p_{m}\right) Q-\delta x \int_{0}^{Q} k\left(Q^{\prime}\right) d Q^{\prime}\right)\right]
$$

In the following subsections we discuss the consequences of this expression in case of either bump or disorder.

$\S$ It can also be regarded as an example of a more general semiclassical perturbation approximation [16] 7. 


\section{1. bump case}

In case of the bump, $k(Q)$ has an amplitude $k_{0}=V_{0} / \hbar v_{E}$ over a spatial scale $\ell$. The total phase variation in (30) is

$$
\delta \phi_{\text {bump }}=\delta x \times k_{0} \ell
$$

This phase variation is in fact the phase space area of the bump. So we have two possibilities: Either $\delta \phi_{\text {bump }} \ll 2 \pi$ or $\delta \phi_{\text {bump }} \gg 2 \pi$. This can be shown to be equivalent to either $\delta x \ll \delta x_{c}^{\text {bump }}$ or $\delta x \gg \delta x_{c}^{\text {bump }}$ respectively. In the former case it is easy to recover the FOPT result (17). One should simply put the perturbation off the exponent, and then do integration by parts. For $n \neq m$ it leads to

$$
P(n \mid m)=\left|\frac{1}{L} \int_{0}^{L} d Q \frac{\mathrm{e}^{-i\left(p_{n}-p_{m}\right) Q}}{p_{n}-p_{m}} \delta x k(Q)\right|^{2}=\delta x^{2}\left|\frac{\mathbf{B}_{n m}}{E_{n}-E_{m}}\right|^{2}
$$

The other possibility $\left(\delta \phi_{\text {bump }} \gg 2 \pi\right)$ guarantees the validity of the standard semiclassical approximation. In such case we cannot put the perturbation off the exponent, but instead we can make a stationary phase approximation. We shall not dwell further on details because it is a standard textbook procedure.

\section{2. disorder case}

We see that for a simple bump we have either FOPT or semiclassical approximation. So we have just two regimes, in spite of the fact that $b \gg 1$. This means that large bandwidth is not a sufficient condition for having three parametric regimes. So let us try to make things more complicated by considering a disordered potential with many bumps. Equation (31) still describes the phase variation over a single bump. This means that $\delta \phi_{\text {bump }} \gg 2 \pi$ is still the relevant condition for a semiclassical approximation! What about first order perturbation theory? The total phase variation in (30) for many bumps is

$$
\delta \phi_{\text {disorder }}=\delta x \times \sqrt{b} \times k_{0} \ell
$$

(note that $b$ is essentially the number of bumps involved). Consequently the validity condition for first order perturbation theory is $\delta \phi_{\text {disorder }} \ll 2 \pi$, which can be easily converted into $\delta x \ll \delta x_{c}^{\text {disorder }}$.

The considerations of the previous paragraph imply that for disordered potential we have three regimes. In the intermediate regime $\delta x_{c} \ll \delta x \ll \delta x_{\mathrm{prt}}$, we have $\delta \phi_{c}^{\text {disorder }} \gg 2 \pi$ while $\delta \phi_{c}^{\text {bump }} \ll 2 \pi$. Consequently we can use neither FOPT, nor the semiclassical approximation. This is the Wigner regime where we expect to find a Lorentzian-like LDOS. Let us demonstrate that indeed a Lorentzian-like LDOS can be obtained from the uniform approximation (30). For this purpose we average $P(n \mid m)$ over realizations of the disorder:

$$
\begin{aligned}
P(n \mid m) & =\frac{1}{L^{2}} \int_{0}^{L} \int_{0}^{L} d Q_{1} d Q_{2} \mathrm{e}^{-i\left(p_{n}-p_{m}\right)\left(Q_{2}-Q_{1}\right)}\left\langle\exp \left[i \delta x \int_{Q_{1}}^{Q_{2}} k\left(Q^{\prime}\right) d Q^{\prime}\right]\right\rangle_{\text {disorder }} \\
& =\frac{1}{L^{2}} \int_{0}^{L} \int_{0}^{L} d Q_{1} d Q_{2} \mathrm{e}^{-i\left(p_{n}-p_{m}\right)\left(Q_{2}-Q_{1}\right)} \exp \left[-\frac{1}{2}\left(\frac{\delta x}{\hbar v_{E}}\right)^{2} \int_{Q_{1}}^{Q_{2}} \int_{Q_{1}}^{Q_{2}}\left\langle V\left(Q^{\prime}\right) V\left(Q^{\prime \prime}\right)\right\rangle d Q^{\prime} d Q^{\prime \prime}\right] \\
& \approx \frac{1}{L} \int_{-\infty}^{\infty} d r \mathrm{e}^{-i\left(p_{n}-p_{m}\right) r} \exp \left[-\delta x^{2}\left(\frac{V_{0}}{\hbar v_{E}}\right)^{2} \ell|r|\right]
\end{aligned}
$$


The last integral is the Fourier transform of an exponential, leading to the Lorentzian LDOS as defined in (18).

\section{Strong localization effect}

Still we have to address the question whether we can trust the uniform approximation, which is based on the WKB wavefunction (29). The answer is known to be negative in case of disordered potential. The WKB approximation does not take into account backscattering, which is responsible for the strong localization effect in 1D disordered potential. It is well known 17 that the localization length is equal (in 1D) to twice the mean free path. Up to a numerical prefactor the Born approximation estimate is

$$
\frac{1}{L_{\mathrm{loc}}} \approx \frac{1}{\hbar v_{E}} \frac{\sigma^{2}}{\Delta} \times \delta x^{2}=\left(\frac{\delta x}{\delta x_{c}}\right)^{2} \frac{1}{L}=\left(\frac{\delta x}{\delta x_{\mathrm{prt}}}\right)^{2} \frac{1}{\ell}
$$

The condition for not being affected by the strong localization effect is $L_{\text {loc }} \gg L$, which leads to $\delta x \ll \delta x_{c}$. Thus it follows that only in the FOPT regime we can ignore the strong localization effect. The strong localization effect cannot be ignored neither in the Wigner regime nor in the semiclassical regime.

The above Born approximation assumes $L_{\mathrm{loc}} \gg \ell$. This condition breaks down if $\delta x>\delta x_{\mathrm{prt}}$. Recall that we also assume that $\delta x$ is small in the classical sense $\left(\delta x V_{0} \ll E\right)$. The two inequalities are consistent if and only if the de Broglie wavelength of the particle is much smaller compared with $\ell$. In this regime we can analyze the localization using the well known transfer matrix approach: Each bump has some transfer matrix, and the random distance between the bumps provides the phase randomization which is assumed in "combining" adjacent transfer matrices. Denoting the average transmission of a "bump" by $g$, one gets

$$
\frac{1}{L_{\mathrm{loc}}} \approx \ln (1 / g) \times \frac{1}{\ell}
$$

Thus even in the $\delta x>\delta x_{\text {prt }}$ regime we can have a very long localization length $\left(L_{\mathrm{loc}} \gg \ell\right)$, which is in fact consistent with the naive expectation.

The implications of the above discussion are, that in spite of the strong localization effect, it is still meaningful to distinguish between three parametric regimes (FOPT, Wigner, semiclassical). We just have to remember that the wavefunction is not ergodic in real space, so the role of $L$ is taken by $L_{\mathrm{loc}}$.

\section{The survival probability $\mathcal{P}(t)$}

We turn to discuss the calculation of the survival probability (1) for the specific "wavepacket dynamics" scenario that has been defined in Section [3. We have the following five strategies of calculation:

- Uniform approximation (which is essentially exact)

- Time domain classical approximation

- Energy domain classical approximation ( $\sim$ LDOS $\leadsto$ Fourier transform)

- Time domain perturbation theory

- Energy domain perturbation theory $(\sim$ LDOS $\leadsto$ Fourier transform)

It can be shown that in typical circumstances the two versions of perturbation theory give in leading order consistent results. This means that we can write a perturbative (essentially first order) result that can be trusted for sufficiently short 
times (for any perturbation $\delta x$ ) or for sufficiently weak perturbation (for any time $t$ ). As an example we consider the deformable harmonic oscillator. Taking equation (15) with appropriate second order compensation of normalization, we get after Fourier transform,

$$
\mathcal{P}^{\text {prt }}(t) \approx 1-2\left(\frac{\delta x}{x}\right)^{2} E^{2} \sin ^{2} t
$$

In a strict time domain FOPT we get only $t^{2}$ time dependence, while in a strict energy domain FOPT we do not get the correct normalization $\left(\mathcal{P}^{\text {prt }}(0)=1\right)$. Still we are able to get one consistent result. The situation is different with the classical approximation. Here time domain and energy domain calculations do not give the same result. As an example we consider again the deformable harmonic oscillator. The calculation of overlap between $\rho_{t}^{c l}$ and $\rho_{0}^{c l}$ is simpler in action-angle variables, but otherwise it is similar to the calculation in Appendix A leading to

$$
\mathcal{P}^{c l}(t)=\left|2 \pi \frac{\delta x}{x} E \sin t\right|^{-1}
$$

The energy domain classical approximation is obtained by squaring the Fourier transform of equation (10) using the classical approximation (13) of $P^{c l}(n \mid m)$, leading to

$$
\mathcal{P}^{c l, E}(t)=\left|J_{0}\left(2 \frac{\delta x}{x} E t\right)\right|^{2}
$$

Although there is no simple exact expression for $\mathcal{P}(t)$, there again exists a very simple uniform approximation which is remarkably accurate in all regimes and therefore we can regard it as "exact," namely

$$
\mathcal{P}^{\text {uniform }}(t)=\left|J_{0}\left(2 \frac{\delta x}{x} E \sin t\right)\right|^{2}
$$

It is derived in Appendix D by using semiclassical expressions for the initial and evolved states, while calculating the overlap exactly rather than by the stationary phase approximation.

Results (37)-(40) are graphically displayed in figure 2 Approximations (37)-(39) can be regarded as various limits of the uniform approximation (40). It is easily seen that (40) reduces to the perturbative result (37) whenever $2(\delta x / x) E \sin (t) \ll 1$. So as expected the perturbative result can be trusted for either small time $t$ or for small perturbation $\delta x / x$. It is also easy to see that the uniform result (40) reduces to the energy domain classical result (39) for $t \ll 1$. The relation of (40) to the time domain classical result (38) is more subtle: For large perturbation the two expressions agree in an asymptotic sense, and either time smoothing or energy averaging is required in order to demonstrate this agreement (see figure 2r).

An important message of this section is that the discussion of regimes in the context of $\mathcal{P}(t)$ is in one to one correspondence with the discussion of LDOS regimes.

\section{Conclusions and final remarks}

In general, for more complicated "wavepacket dynamics" scenarios, which may involve time dependent Hamiltonian, an explicit reduction of the survival probability problem to LDOS study is not possible [6]. The simplest way to make the Hamiltonian "time 

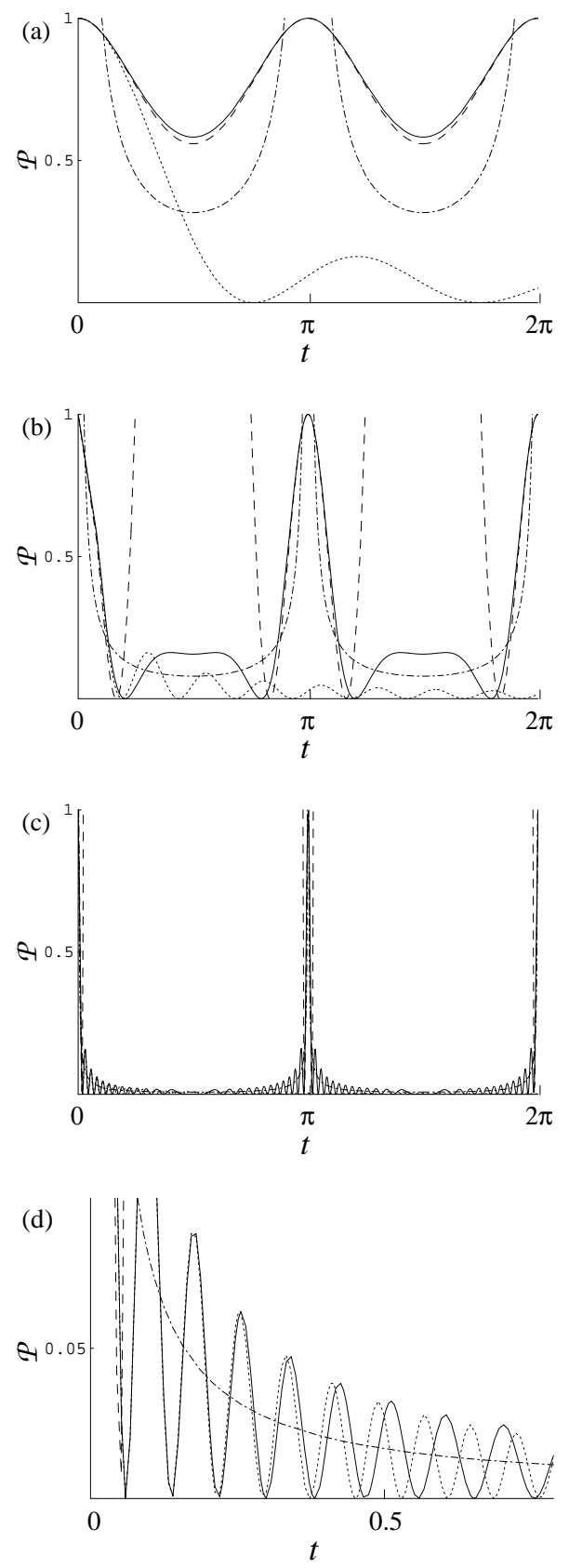

Figure 2. The survival probability, using the same parameters as in figure 1 (a) Perturbative regime. (b) Intermediate regime. (c) Semiclassical regime. In each panel the solid line is the uniform result 40], the dashed line is the perturbative result (37), the dashed-dotted line is the time domain classical result (38), and the dotted line is the energy domain classical result (39). Panel (d) gives a short-time detail of panel (c). 
dependent" is by changing it either once (as in "fidelity" or "Loschmidt echo" studies) or repeatedly (as in kicked systems). The latter possibility opens the way for "chaos" in the classical dynamics of one-dimensional (1D) systems. A prototype system for 1D chaos studies is the kicked rotator: This is a particle in a ring, which is periodically kicked by a cosine potential. As in the standard formulation of "wavepacket dynamics" it is common to assume an initial preparation which is supported by a manifold $P=p_{m}$. As a result of the kicks the initial manifold becomes extremely convoluted. If we want to calculate $\mathcal{P}(t)$ we have to trace the evolving manifold with the initial one. The calculation of this overlap can be carried out using the uniform approximation that we have discussed previously [12, 13, 7].

There is one major difference that makes the "chaotic" scenario that we have described above different from the LDOS calculation: The issue of strong localization, which has been discussed in Section 9 is no longer relevant. The evolving state is "distributed" over the whole manifold $\|$.

In view of the above, the calculations that we have presented in this paper, using the uniform approximation, are in fact generic. In particular we have explained how a Fermi-golden-rule behavior arises within this framework. A refinement of the uniform approximation using the "replacement manifolds" approach has been introduced in references [12, 13].

The reader can be tempted to reach the conclusion that the discussion of "regimes" within the framework of the "uniform approximation" can be trivially generalized from $d=1$ chaotic systems to $d>1$ chaotic systems. This is in fact not quite correct. In order to explain the difficulty let us consider again LDOS calculation. In one-dimensional systems what we have to do is to calculate the overlap of two onedimensional manifolds. We can do this calculation semiclassically if we can trust the stationary phase approximation. This leads to the " $\hbar$ area" condition: the stationary phase approximation is accurate if the phase space area delimited by the two manifolds between two stationary phase points is larger than $\hbar$. What is the generalization of this condition in the $d>1$ case? Now the manifolds are "surfaces" and they intersect along "lines". So the concept of "stationary points" becomes inapplicable, and also the " $\hbar$ area" condition becomes meaningless. The way out of this difficulty [10] is to use the Wigner function point of view. Then one realizes that the proper way to formulate the semiclassical condition is to say that the separation between the surfaces should be much larger compared with the "thickness" of Wigner function. This "thickness" is just the "bandwidth" that we have discussed in this Paper.

Conventionally, semiclassical methods are applied in the "semiclassical" regime (large enough perturbation). In the perturbative regime people use perturbation theory. A major motivation for the present line of study is to extend the applicability of semiclassical methods into the perturbative regime. Most of the calculations that we have presented under the heading "uniform approximation" can be reformulated using the Wigner function language. This opens the way towards a unified semiclassical understanding of "regimes" in case of $d>1$ systems.

\| The only reservation for this statement is the possibility of witnessing a "dynamical localization effect" after an extremely long time (known as the "breaktime"). For small $\hbar$ the existence of a "breaktime" will have a negligible effect on the behavior of $\mathcal{P}(t)$. In fact we should remember that also in non-kicked systems we have a "breaktime", which is just the Heisenberg time. We also note that this type of localization is apparently captured by semiclassical methods [18] 


\section{Acknowledgments}

It is our pleasure to thank Bilha Segev (BGU) and Eric Heller (Harvard) for useful discussions. The research was supported by the Institute for Theoretical Atomic and Molecular Physics at Harvard University, by the Mathematical Sciences Research Institute at Berkeley, and by the Israel Science Foundation (grant No.11/02).

\section{Appendix A. Calculation of $P^{c l}(n \mid m)$ and $P^{\text {exact }}(n \mid m)$}

We shall demonstrate in this appendix the calculation of $P^{c l}(n \mid m)$ for the deformable harmonic oscillator:

$$
\begin{aligned}
P^{c l}(n \mid m) & =\int \frac{d Q d P}{2 \pi} \delta\left[H\left(Q, P ; x_{2}\right)-E_{n}\right] \delta\left[H\left(Q, P ; x_{1}\right)-E_{m}\right] \\
& =\int \frac{d Q}{2 \pi} \sum_{P= \pm \sqrt{2 E_{m}-\left(Q / x_{1}\right)^{2}} / x_{1}} \frac{\delta\left[\mathcal{H}(Q, P ; x)-E_{n}\right]}{x_{1}^{2}|P|} \\
& =\frac{1}{\pi} \int d Q \frac{\delta[f(Q)]}{\sqrt{2 x_{1}^{2} E_{m}-Q^{2}}} \\
& =\frac{1}{\pi} \sum_{ \pm}\left(2 x_{1}^{2} E_{m}-Q_{ \pm}^{2}\right)^{-1 / 2}\left|f^{\prime}\left(Q_{ \pm}\right)\right|^{-1} \\
& =\frac{1}{\pi} \frac{x_{1} x_{2}}{\sqrt{\left(x_{2}^{2} E_{n}-x_{1}^{2} E_{m}\right)\left(x_{2}^{2} E_{m}-x_{1}^{2} E_{n}\right)}}
\end{aligned}
$$

where

$$
f(Q) \equiv \frac{x_{2}^{4}-x_{1}^{4}}{2 x_{1}^{4} x_{2}^{2}} Q^{2}+\left(\frac{x_{2}}{x_{1}}\right)^{2} E_{m}-E_{n} .
$$

and $Q_{ \pm}$are the roots of the equation $f(Q)=0$,

$$
Q_{ \pm}= \pm x_{1} x_{2} \sqrt{\frac{2\left(x_{2}^{2} E_{m}-x_{1}^{2} E_{n}\right)}{x_{2}^{4}-x_{1}^{4}}}
$$

for which

$$
f^{\prime}\left(Q_{ \pm}\right)= \pm x_{1}^{-3} x_{2}^{-1} \sqrt{2\left(x_{2}^{2} E_{m}-x_{1}^{2} E_{n}\right)\left(x_{2}^{4}-x_{1}^{4}\right)}
$$

Equation (13) is a simplified version of this result, assuming that $\delta x \ll x$.

It is also possible to obtain an exact result in the quantum mechanical case. The explicit expression for the eigenfunction using Hermite polynomials is

$$
\langle Q \mid n(x)\rangle=\left(\pi x^{2}\right)^{-1 / 4}\left(2^{n} n !\right)^{-1 / 2} H_{n}(Q / x) \mathrm{e}^{-(Q / x)^{2} / 2}
$$

This leads to

$$
\begin{aligned}
& \left\langle n\left(x_{2}\right) \mid m\left(x_{1}\right)\right\rangle=\left(\pi x_{1} x_{2}\right)^{-1 / 2}\left(2^{n+m} n ! m !\right)^{-1 / 2} \\
& \quad \times \int_{-\infty}^{\infty} d Q H_{n}\left(Q / x_{2}\right) H_{m}\left(Q / x_{1}\right) \mathrm{e}^{-\frac{1}{2}\left(x_{1}^{-2}+x_{2}^{-2}\right) Q^{2}}
\end{aligned}
$$

Upon squaring one obtains $P(n \mid m)$. The integral in (A.6) becomes highly oscillatory for high-lying eigenstates in which we are interested, and numerical calculation is tricky. To our surprise, this intimidating integral can be evaluated analytically even for $x_{1} \neq x_{2}$, resulting in a finite sum of terms, which is not very elegant, but very simple 
to evaluate on a computer. For the exact benchmark in the numerical results presented in this paper, we therefore used this analytical expression instead of numerically evaluating the integral A.6.

Note that both classically and quantum mechanically the overlap $P(n \mid m)$ depends only on the ratio $x_{2} / x_{1}$. In the classical case $n$ is a real index $\left(E_{n}\right.$ can have any real positive value). In the quantum mechanical case $n$ is an integer index $\left(E_{n}=n+\frac{1}{2}\right)$. Due to the reflection symmetry of the Hamiltonian there is an overlap only between states with the same parity. The overlap for $|n-m|=$ odd vanishes. Whenever we say that $P(n \mid m) \approx P^{c l}(n \mid m)$, it should be interpreted in a coarse grain sense. Therefore, for sake of graphical presentation we have plotted $P(n \mid m)$ versus $P^{c l}(n \mid m) / 2$. One may say that the plotted $P(n \mid m)$ and $P^{c l}(n \mid m) / 2$ correspond to a de-symmetrized oscillator.

\section{Appendix B. Calculation of $P^{\text {prt }}(n \mid m)$}

We shall demonstrate in this appendix the calculation of $P^{p r t}(n \mid m)$ for the deformable harmonic oscillator. From first order perturbation theory we know that for $m \neq n$

$$
\left\langle m\left(x_{0}\right) \mid n\left(x_{0}+\delta x\right)\right\rangle \approx \frac{\langle m|\delta \mathcal{H}| n\rangle}{E_{n}-E_{m}}
$$

Using

$$
\left\langle m\left|Q^{2}\right| n\right\rangle,\left\langle m\left|P^{2}\right| n\right\rangle=\left(\frac{1}{2}+n\right) \delta_{m n} \pm \frac{1}{2}\left[\sqrt{(n+1)(n+2)} \delta_{m, n+2}+\sqrt{n(n-1)} \delta_{m, n-2}\right]
$$

one obtains

$$
P^{\mathrm{prt}}(n \mid m) \approx \delta_{n, m}+\frac{1}{4}\left(\frac{\delta x}{x}\right)^{2}\left(E^{2}-\frac{1}{4}\right) \delta_{|n-m|, 2}
$$

where $E=\left(E_{n}+E_{m}\right) / 2$. In the text we have presented a simplified version that assumes $E \gg 1$.

\section{Appendix C. Calculation of $P^{\text {uniform }}(n \mid m)$}

The following canonical transformation is used in order to transform the Hamiltonian of the harmonic oscillator to action-angle variables:

$$
\begin{aligned}
& Q=\sqrt{2 I} \cos (\phi) \\
& P=\sqrt{2 I} \sin (\phi)
\end{aligned}
$$

In the vicinity of $x_{0}=1$ it leads to

$$
\begin{aligned}
\mathcal{H}(\phi, I ; x) & =I\left[(1 / x) \cos ^{2} \phi+x \sin ^{2} \phi\right] \\
& \approx I-\delta x I \cos (2 \phi))+\mathcal{O}\left(\delta x^{2}\right)
\end{aligned}
$$

The canonical transformation from $(\phi, I)$ to the action angle variables $\left(\phi^{\prime}, I^{\prime}\right)$ of the perturbed Hamiltonian is derived from a generating function $S\left(\phi, I^{\prime}\right)$. The manifold $I^{\prime}=$ const is determined from the equation $\mathcal{H}(\phi, I ; x)=$ const, leading to the relation $I=[1+\delta x \cos (2 \phi)] I^{\prime}$. The generating function should satisfy $I=\partial S / \partial \phi$. Therefore one deduces that

$$
S\left(\phi, I^{\prime}\right)=[\phi+\delta x \sin (2 \phi)] I^{\prime}
$$


The semiclassical expression for the wavefunction is

$$
\left\langle\phi \mid I^{\prime}\right\rangle_{\mathrm{sc}}=(2 \pi)^{-1 / 2} \sqrt{\frac{\partial^{2} S}{\partial I^{\prime} \partial \phi}} \mathrm{e}^{i S\left(\phi, I^{\prime}\right)} .
$$

The $n$th semiclassical eigenstate corresponds to the substitution $I^{\prime}=E_{n}=n+\frac{1}{2}$. For technical simplicity it is convenient to calculate the overlap between two perturbed wavefunctions $\left(x=x_{0} \pm \delta x / 2\right)$.

$$
\begin{aligned}
& \left\langle n\left(x_{0}+\delta x / 2\right) \mid m\left(x_{0}-\delta x / 2\right)\right\rangle=\int_{0}^{2 \pi} d \phi\left\langle\phi \mid I^{\prime \prime}=E_{n}\right\rangle^{*}\left\langle\phi \mid I^{\prime}=E_{m}\right\rangle \\
& =\frac{1}{2 \pi} \int_{0}^{2 \pi} d \phi \sqrt{1-\delta x^{2} \cos ^{2} 2 \phi} \times \exp \left[i\left(E_{m}-E_{n}\right) \phi-i \delta x E \sin 2 \phi\right] \\
& \approx J_{(m-n) / 2}(\delta x E)
\end{aligned}
$$

Above $I^{\prime}$ and $I^{\prime \prime}$ correspond to the perturbations $\pm \delta x / 2$, and $E=\left(E_{n}+E_{m}\right) / 2$. In the main text we have reverted to a more general version of this expression that does not assume $x_{0}=1$. It is important to realize that because of the assumption $\delta x \ll x$ the pre-exponential factor (which is in fact a "classical" factor) can be neglected in leading order. Figure 1 confirms the remarkable agreement of the uniform approximation with the exact result.

\section{Appendix D. Calculation of $\mathcal{P}^{\text {uniform }}(t)$}

For the purpose of calculation it is more convenient to regard the prepared state as a "perturbed state" and the evolution Hamiltonian as the "unperturbed Hamiltonian". This is of course equivalent to the presentation in the text upon the replacement $\delta x \mapsto-\delta x$.

The initial state $\left|m\left(x_{0}+\delta x\right)\right\rangle$ is characterized by the action $I^{\prime}=E_{m} \equiv E$. It is represented as in Appendix C by

$$
\left\langle\phi \mid I^{\prime}=E\right\rangle \approx \frac{1}{2 \pi} \exp [i(\phi+\delta x \sin 2 \phi) E]
$$

The evolving state is represented by

$$
\left\langle\phi\left|\mathrm{e}^{-i t \mathcal{H}_{0}}\right| I^{\prime}=E\right\rangle=\left\langle\phi-t \mid I^{\prime}=E\right\rangle
$$

The overlap between the evolving and the initial state is

$$
\frac{1}{2 \pi} \int_{0}^{2 \pi} d \phi \exp [i(-t+\delta x[\sin 2(\phi-t)-\sin 2 \phi]) E]
$$

For $F(t)$ as defined in (12) we obtain (after the required replacement $\delta x \mapsto-\delta x$ ):

$$
F(t)=\frac{1}{2 \pi} \int_{0}^{2 \pi} d \phi \exp [i \delta x E \sin t \cos (2 \phi-t)]=J_{0}(2 \delta x E \sin t)
$$

The survival probability is obtained by squaring this result.

\section{References}

[1] Cohen D and Heller E J 2000 Phys. Rev. Lett. 842841

[2] Heller E J 1991 Chaos and Quantum Systems, ed M-J Giannoni et al (Amsterdam: Elsevier)

[3] Kallush S, Segev B, Sergeev A V and Heller E J 2002 J. Phys. Chem. A 1066006

Sergeev A V and Segev B 2002 J. Phys. A: Math. Gen. 351769 
[4] Jalabert R A and Pastawski H M 2001 Phys. Rev. Lett. 862490

Cucchietti F M, Pastawski H M, Jalabert R 2000 Physica A 283285

J.V. Emerson, Phys. Rev. Lett. 89, 284102 (2002).

Cucchietti F M, Pastawski H M and Wisniacki D A 2002 Phys. Rev. E 65045206

Cucchietti F M, Lewenkopf H, Mucciolo E R, Pastawski H M and Vallejos R O 2002 Phys. Rev. E 65046209

Benenti G and Casati G 2002 Phys. Rev. E 65066205

Cerruti N R and Tomsovic S Phys. Rev. Lett. 88054103

Prosen T 2001 Preprint quant-ph/0106149

Prosen T and Znidaric M 2001 J. Phys. A 34 L681

Eckhardt B 2003 J.Phys. A 36371

Silvestrov P G, Tworzydlo J and Beenakker C W J 2002 Preprint nlin.CD/0207002

[5] Jacquod P, Silvestrov P G and Beenakker C W J 2001 Phys. Rev. E 64055203

[6] Wisniacki D A and Cohen D 2002 Phys. Rev. E 66046209

[7] Vaníček J and Heller E J 2003 Preprint quant-ph/0302192

[8] For a pedagogical presentation, including references, see references [9] and [10, which can be downloaded from http://www.bgu.ac.il/ dcohen

[9] Cohen D 2002 "Driven chaotic mesoscopic systems, dissipation and decoherence" in Dynamics of Dissipation: Proceedings of the 38th Karpacz Winter School of Theoretical Physics ed P Garbaczewski and R Olkiewicz (Springer)

[10] Cohen D 2000 "Chaos, dissipation and quantal Brownian motion" in New Directions in Quantum Chaos: Proceedings of the International School of Physics Enrico Fermi Course CXLIII ed G Casati, I Guarneri and U Smilansky (Amsterdam: IOS Press)

[11] Cohen D 1999 Phys. Rev. Lett. 824951

[12] Vaníček J and Heller E J 2001 Phys. Rev. E 64026215

[13] Vaníček J and Heller E J 2003 Phys. Rev. E 67016211

[14] Cohen D and Kottos T 2001 Phys. Rev. E 6336203

[15] Miller W H 1974 Adv. Chem. Phys. 2569

[16] Miller W H and Smith F T 1978 Phys. Rev. A 17939

[17] Richter K, Ullmo D and Jalabert R A 1996 Phys. Rev. B 54 R5219

[18] L. Kaplan, Phys. Rev. Lett. 81, 3371 (1998). 